\title{
Rapid-Deployment Aortic Valve Replacement after Previous Mechanical Valve Implantation
}

\author{
Markus Schlömicher ${ }^{1}$ Peter Lukas Haldenwang ${ }^{1}$ Vadim Moustafine $^{1} \quad$ Britta Wolf $^{2}$ Peter Zahn ${ }^{2}$ \\ Matthias Bechtel ${ }^{1}$ Justus Thomas Strauch ${ }^{1}$ \\ ${ }^{1}$ Department of Cardiothoracic Surgery, Ruhr University Hospital \\ Bergmannsheil - Bochum, North Rhine-Westphalia, Germany \\ 2 Department of Anesthesiology, Ruhr University Hospital \\ Bergmannsheil - Bochum, North Rhine-Westphalia, Germany \\ Address for correspondence Markus Schlömicher, MD, Department of \\ Cardiothoracic Surgery, Ruhr University Hospital Bergmannsheil, \\ Bürkle de la Camp Platz 1, 44789 Bochum, North Rhine-Westphalia, \\ Germany (e-mail: m.schloemicher@gmail.com).
}

Thorac Cardiovasc Surg Rep 2016;5:24-26.

\begin{abstract}
Keywords

- aortic valve

- aortic root

- reoperation

- valve surgery

Recent studies report a reproducible reduction of myocardial ischemic and cardiopulmonary bypass times along with excellent hemodynamics and low rates of paravalvular leakage for rapid-deployment valves. A 68-year-old female patient with aortic stenosis and a mechanical mitral valve which was implanted in 2006 received rapid-deployment aortic valve replacement. The procedure could be performed with a cross-clamp time of 45 minutes and a cardiopulmonary bypass time of 60 minutes. Postoperative course was uncomplicated and the patient was discharged to the referring hospital on postoperative day 8.
\end{abstract}

\section{Introduction}

Recent studies report a reproducible reduction of myocardial ischemic and cardiopulmonary bypass times along with excellent hemodynamics and low rates of paravalvular leakage for rapid-deployment valves. ${ }^{1,2}$ Especially minimal access surgery can be simplified and accelerated. ${ }^{1}$ Expanding the field of commitment to combined and complex procedures is a subsequent step following this perception. The Edwards INTUITY Elite valve (Edwards Lifesciences; Irvine, California, United States) is a stented, trileaflet, bovine pericardial bioprosthesis based on the Carpentier-Edwards PERIMOUNT platform, mounted on a balloon-expandable stent frame, which is covered by a polyester sealing cloth, anchoring the valve in the outflow tract after balloon inflation.

\section{Technique}

A 68-year-old female patient underwent mechanical mitral valve replacement (MVR) in 2006 using Medtronic Hall prosthesis (Medtronic; Minneapolis, Minnesota, United States) with a tilting disc mechanism.

received

November 13, 2015

accepted after revision

January 15, 2016

published online

April 4, 2016 10.1055/s-0036-1579681. ISSN 2194-7635.
In 2015 , the patient newly presented with severe cardiac decompensation. Transthoracic echocardiogram and transesophageal echocardiogram (TEE) examination could show a combined aortic vitium with severe aortic stenosis and moderate aortic insufficiency. An aortic valve area of $0.8 \mathrm{~cm}^{2}$ was measured along with pressure half-time of $161 \mathrm{~ms}$ and a vena contracta of $5 \mathrm{~mm}$. No echocardiographic evidence of prosthesis malfunction was found so that primarily the indication for aortic valve replacement (AVR) in a redo setting was established. The distance between the aortic and the mitral annulus was measured to be $9 \mathrm{~mm}$ (-Fig. $\mathbf{1}$ ).

The procedure was performed using a standard median sternotomy with direct cannulation of the ascending aorta and the right atrial appendage. Cold blood cardioplegia was administered in an antegrade fashion, and a vent was inserted in the right upper pulmonary vein. Cardioplegia was readministered in a 20-minute interval.

A hockey-stick aortotomy was performed to expose the aortic valve, which showed a heavily calcified tricuspid native valve. The mitral prosthesis was inspected endoscopically after the excision of the aortic leaflets and decalcification of the annulus. Extensive calcification of the mitral annulus
License terms

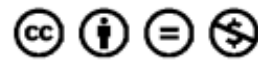

(c) 2016 Georg Thieme Verlag KG Stuttgart · New York 


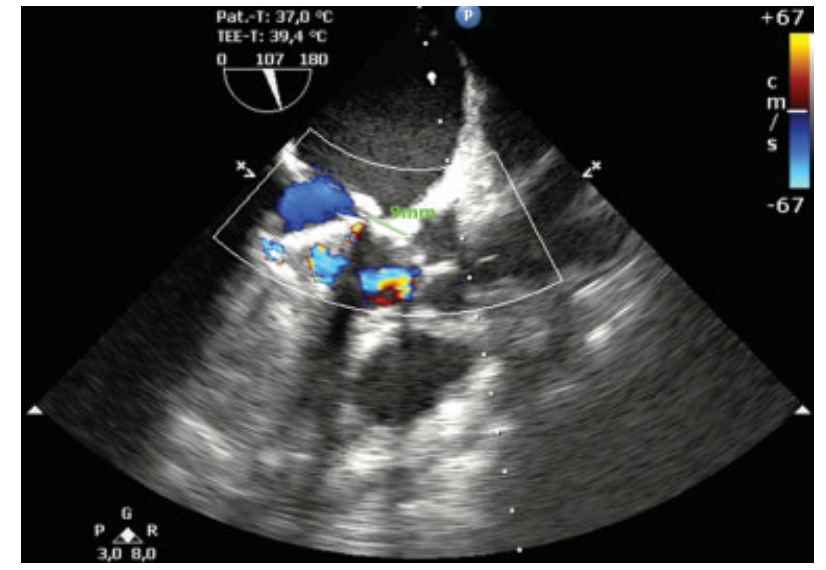

Fig. 1 Preoperative TEE illustrating the distance between the mitral prostheses and the aortic annulus.

could be found but no thrombus or pannus formation potentially impeding the hinge mechanism.

Extensive attention was paid to the sizing, especially the relation of the sizer inlet-which represents the height of polyester-covered stent frame-to the mitral prosthesis. No interference with the sewing and the tilting disc was seen.

After the sizing procedure, three guiding sutures were placed at the nadir of each aortic sinus.

Braided, nonpledged, double-armed sutures that were stitched through the annulus in U-stitch fashion, and through three marked positions on the sewing cuff, were used. Then the valve with the attached delivery system was lowered into place in the annulus under direct vision, with the guiding sutures being snared with a tourniquet. Finally, the balloon catheter was inserted through the holding device and locked into place. The balloon was inflated using saline for a 10 -second time period with a pressure of 4.5 atmospheres. The sutures were tied after removal of the entire holding device. Closure of the aortotomy was performed in a typical double-layer fashion.

The procedure could be performed with a cross-clamp time of 45 minutes and a cardiopulmonary bypass time of 60 minutes.

Intraoperative TEE could not detect any paravalvular leakage (-Fig. 2).

The postoperative course was uneventful. The patient could be extubated after a ventilation time of 10 hours. No bleeding occurred. The patient could be transferred to ward on postoperative day (POD) 2. The further course was completely uneventful so that the patient was discharged to the referring hospital on POD 8 (-Fig. 3). A telephone follow-up was done 6 months after the operation. No adverse events occurred in this period.

\section{Discussion}

Redo surgery in patients with mechanical prostheses in mitral position requiring AVR represents a challenge for the surgeon. Although a hospital mortality of only $5.1 \%$ has been recently published in the RECORD (REdo Cardiac Operation Research Database) study, cardiopulmonary bypass and cross-clamp times were identified as independent predictors for early and late mortality. ${ }^{3}$ Therefore, the acceleration of the implant process

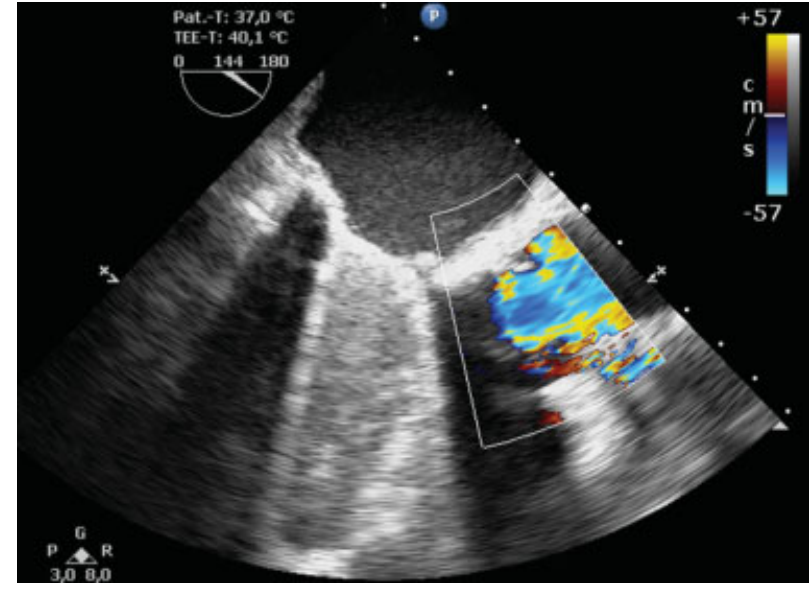

Fig. 2 Intraoperative TEE after implantation of the rapid-deployment valve showing no paravalvular leakage.

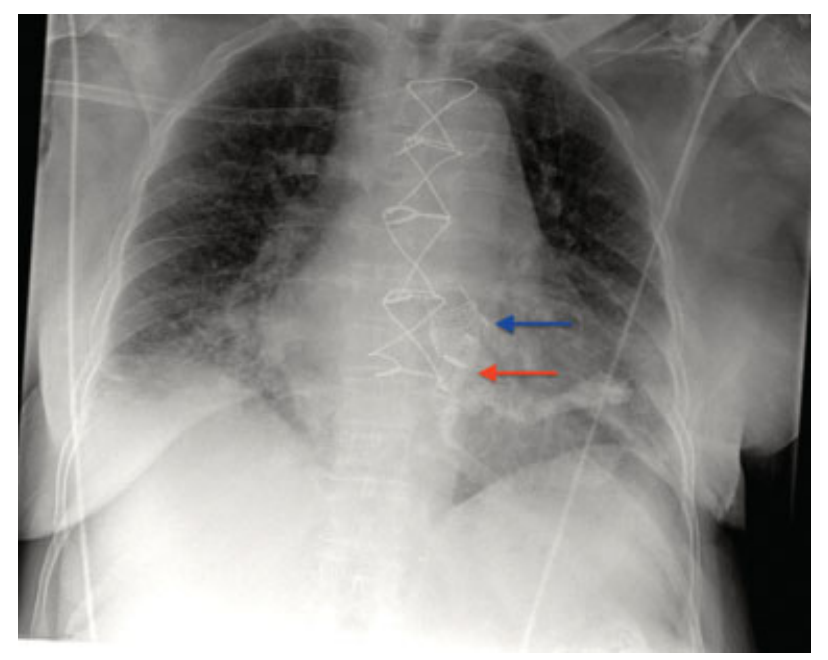

Fig. 3 Postoperative X-ray after the implantation of a rapid-deployment valve. The red arrow indicates the mechanical prosthesis in mitral position and the blue arrow the rapid-deployment valve in aortic position.

offered by the Edwards INTUITY Elite valve system might be beneficial especially in redo scenarios. However, no case of rapid-deployment AVR with the Edwards INTUITY Elite valve system in patients with mechanical prostheses has been described so far, whereas the Perceval valve system (Sorin Biomedica Cardio Srl; Saluggia, Italy)-which represents a sutureless system-was successfully implanted in a redo operation after previous mechanical MVR. ${ }^{4}$

The use of the Edwards INTUITY Elite valve system is not contraindicated in the setting of concomitant mitral valve procedures or preexisting valves in mitral position according to the instructions for use given by the manufacturer. However, strong doubts exist that a prosthesis in mitral position could alter the three-dimensional structure of the aortic root and the left ventricular outflow tract or directly interfere with the balloon-expandable stent of the valve system. Nevertheless, several publications show the feasibility of transcatheter aortic procedures after previous mechanical or biological MVR. An increase in paravalvular leaks, high gradients, or 
valve distortion was not measurable. ${ }^{5-7}$ Given that rapiddeployment valves-as well as devices used in transcatheter aortic valve implantation (TAVI) procedures-have a similar anchoring mechanism through an expandable stent frame, reproducible results should be achieved in rapid-deployment AVR. Beller et al suggest a minimal distance of $10 \mathrm{~mm}$ between the aortic and the mitral annulus for TAVI procedures in the presence of a mechanical mitral prosthesis. ${ }^{7}$ In fact, in our case a distance of $9 \mathrm{~mm}$ was measured (-Fig. 1).

Ferrari et al could demonstrate the successful use of the Edwards INTUITY valve system in concomitant aortic and mitral valve surgery. ${ }^{8}$ No interference between the INTUITY valve and either a mitral bioprostheses or a mitral ring was seen in this case series. The height of the subannular balloon-expandable stent frame is 6 to $8 \mathrm{~mm}$ depending on the valve size. Therefore, impediment of the tilting disc mechanism was not expected. The only limiting factor would be a possible interference with the sewing ring of the mitral prostheses. A simple simulation of the final valve position using the inlet of the INTUITY sizer, which represents the balloon-expandable stent frame in the outflow tract, can reveal this problem. In case of interference, we would normally have chosen to implant a conventional pericardial biological valve, despite the existing mechanical valve in mitral position, because of the advanced patient's age. A high incidence of severe paravalvular leakage or high transvalvular gradients resulting from suboptimal subannular expansion of the polyester-covered stent frame might occur.

This case demonstrates the feasibility of a rapid-deployment procedure in a redo setting after previous mechanical MVR along with a sufficient reduction of myocardial ischemic and cardiopulmonary bypass times.

\section{References}

1 Schlömicher M, Haldenwang PL, Moustafine V, Bechtel M, Strauch JT. Minimal access rapid deployment aortic valve replacement: initial single-center experience and 12-month outcomes. J Thorac Cardiovasc Surg 2015;149(2):434-440

2 Kocher AA, Laufer G, Haverich A, et al. One-year outcomes of the Surgical Treatment of Aortic Stenosis With a Next Generation Surgical Aortic Valve (TRITON) trial: a prospective multicenter study of rapid-deployment aortic valve replacement with the EDWARDS INTUITY valve system. J Thorac Cardiovasc Surg 2013;145(1):110-115, discussion 115-116

3 Onorati F, Biancari F, De Feo M, et al. Mid-term results of aortic valve surgery in redo scenarios in the current practice: results from the multicentre European RECORD (REdo Cardiac Operation Research Database) initiative. Eur J Cardiothorac Surg 2015;47(2): 269-280, discussion 280

4 Mazine A, Minh TH, Bouchard D, Demers P. Sutureless aortic valve replacement in the presence of a mechanical mitral prosthesis. J Thorac Cardiovasc Surg 2013;146(4): e27-e28

5 Soon JL, Ye J, Lichtenstein SV, Wood D, Webb JG, Cheung A. Transapical transcatheter aortic valve implantation in the presence of a mitral prosthesis. J Am Coll Cardiol 2011;58(7): 715-721

6 Schlömicher M, Haldenwang PL, Moustafine V, Bechtel M, Strauch JT. Simultaneous double valve-in-valve TAVI procedure for failed bioprostheses. Ann Thorac Surg 2015; 99(2):722-724

7 Beller CJ, Bekeredjian R, Krumsdorf U, et al. Transcatheter aortic valve implantation after previous mechanical mitral valve replacement: expanding indications? Heart Surg Forum 2011;14(3): E166-E170

8 Ferrari E, Siniscalchi G, Marinakis S, Berdajs D, von Segesser L. 'Fast-implantable' aortic valve implantation and concomitant mitral procedures. Interact Cardiovasc Thorac Surg 2014;19(4): 682-684 